\title{
Osteoprotegerin promotes the proliferation of chondrocytes and affects the expression of ADAMTS-5 and TIMP-4 through MEK/ERK signaling
}

\author{
ZHI-YUN FENG ${ }^{1 *}$, ZHEN-NIAN HE $^{2 *}$, BIN ZHANG $^{2}$ and ZHONG CHEN ${ }^{1}$ \\ ${ }^{1}$ Department of Orthopedics, The First Affiliated Hospital, College of Medicine, Zhejiang University, Hangzhou, \\ Zhejiang 310003; ${ }^{2}$ Department of Orthopedics, Beilun People's Hospital, Ningbo, Zhejiang 315806, P.R. China
}

Received April 19, 2013; Accepted September 26, 2013

DOI: $10.3892 / \mathrm{mmr} .2013 .1717$

\begin{abstract}
The involvement of osteoprotegerin (OPG) in bone metabolism has previously been established; however, whether OPG regulates chondrocytes directly and exerts precise cellular and molecular effects on chondrocytes remains to be determined. Thus, the present study aimed to investigate the direct effect of OPG on the viability, proliferation and functional consequences of chondrocytes. Primary chondrocytes were isolated from the knee of Sprague-Dawley rats. Passage 1 chondrocytes were identified by toluidine blue staining and used in the experiments. The cell proliferation induced by OPG at various concentrations was measured by a Cell Counting kit-8 (CCK-8) assay. Following pretreatment with mitogen-activated/extracellular signal-regulated kinase kinase (MEK) inhibitor U0126, extracellular signal-regulated kinase (ERK) inhibitor PD098059, and P38 mitogen-activated protein kinase (P38MAPK) inhibitor SB203580 for $30 \mathrm{~min}$, chondrocytes were treated with OPG, and CCK-8 was performed. The cellular signals of MAPKs, including ERK, P38MAPK and c-Jun N-terminal protein kinase (JNK), were investigated by western blot analysis following treatment with OPG. The functional consequences following treatment with soluble OPG were analyzed by qPCR and western blot analysis. OPG increased chondrocyte proliferation with maximal effect at $10 \mathrm{ng} / \mathrm{ml}$, and induced the phosphorylation of MEK and ERK but not P38MAPK or JNK. Suppression of ERK activity via PD098095 inhibited OPG-induced chondrocyte proliferation. Administration of OPG significantly downregulated ADAMTS-5 and upregulated tissue inhibitor
\end{abstract}

Correspondence to: Professor Zhong Chen, Department of Orthopedics, The First Affiliated Hospital, College of Medicine, Zhejiang University, 79 Qingchun Road, Hangzhou, Zhejiang 310003, P.R. China

E-mail: chenzhong456@yahoo.cn

"Contributed equally

Key words: osteoprotegerin, proliferation, chondrocyte, mitogen-activated/extracellular signal-regulated kinase kinase, ADAMTS-5, tissue inhibitor of metalloproteinase-4 of metalloproteinase (TIMP)-4 production, but had no effect on the expression of TIMP-1, -2 and -3 , insulin-like growth factor I, transforming growth factor- $\beta$, basic fibroblast growth factor, bone morphogenetic protein-2, collagen II, aggrecan and ADAMTS-4. Suppression of ERK activity via PD098095 inhibited the alteration of ADAMTS-5 and TIMP-4 expression induced by OPG. OPG therefore regulated the proliferation of chondrocytes via MEK/ERK signaling, and directly affected chondrocytes by influencing the expression profile of ADAMTS-5 and TIMP-4.

\section{Introduction}

It has been established that the osteoprotegerin (OPG)/RANKL/RANK system is important in bone metabolism and remodeling. The receptor activator of nuclear factor (NF)- $\kappa$ B ligand, RANKL, is localized or expressed by osteoblasts and binds to its receptor RANK, which is localized or expressed by osteoclasts, leading to the enhancement of osteoclastogenesis. OPG suppresses osteoclastogenesis via binding to RANKL. In addition to its involvement in bone metabolism, OPG has been implicated in the vascular system and in cartilage metabolism (1-5). It has been suggested that OPG is involved in cartilage, as OPG almost completely inhibited cartilage loss in an adjuvant-induced arthritis (AIA) rat model (6). In addition, Shimizu et al (5) demonstrated that OPG-deficient mice exhibited thinning of the articular cartilage layers, with superficial fibrillation and proteoglycan defects. Intraarticular or systemic OPG treatment has been demonstrated to protect articular cartilage from proteoglycan depletion and destruction of the cartilage surface $(5,7)$. However, the precise mechanism of OPG protection of cartilage remains largely unknown. Shimizu et al (5) hypothesized that OPG protected cartilage by inhibiting the apoptosis of chondrocytes, as a direct effect. By contrast, findings of another study (2) suggested that the protection effect of OPG was obtained via subchondral bone protection. In in vitro studies, which added exogenous RANK1 to the supernatant of cultured chondrocytes, it was observed that $\mathrm{NF}-\kappa \mathrm{B}$ was not activated, the expression profile of genes encoding proinflammatory mediators in chondrocytes was not altered and this effect was also observed for the production of collagenase and nitric oxide (3). These results suggest that OPG 
exerts no effect on chondrocytes as it is currently understood that OPG exerts its functions by counteracting RANKL. Kwan Tat et al (8) demonstrated that exogenous OPG-Fc (with an Fc domain) increased the production of two catabolic factors, matrix metalloproteinase (MMP)-13 and protease-activated receptor 2 (PAR-2). This finding suggested that OPG exerts a direct effect on cartilage. In studies concerning the involvement of OPG in other systems, Yongchaitrakul et al (9) observed that OPG increased osteopontin production via sydecan-1. Kobayashi-Sakamoto et al (1) demonstrated that OPG promoted the proliferation of human dermal microvascular endothelial cells, and was protective from cell death induced by Porphyromonas gingivalis, a pathogen which causes adult periodontitis. Thus this study aimed to determine whether OPG exerts a direct effect on chondrocytes.

Cartilage matrices are predominantly composed of a fibrillar collagen network and aggregated proteoglycan (10). Chondrocytes are the only cell elements that are responsible for the metabolism of cartilage. In normal conditions, chondrocytes produce and maintain the cartilaginous matrix, self-secrete several factors and create a balanced local environment regulating the metabolism of cartilage. Briefly, catabolic factors such as MMP-1, -3, -9 and -13, or aggrecanase-1 and -2 (ADAMTS-4, -5) produced by chondrocytes degrade the matrix of cartilage. Conversely these factors are also able to increase the secretion of tissue inhibitor of metalloproteinase (TIMP)-1, -2, -3, -4, insulin-like growth factor (IGF)-I, transforming growth factor (TGF)- $\beta$, bone morphogenetic protein (BMP)-2 and basic fibroblast growth factor (bFGF) to inhibit the effect of MMPs, and reach a balance to maintain the selfrenewal of cartilage. Moreover, IGF-I, TGF- $\beta$, BMP-2 and $\mathrm{bFGF}$ are predominant factors in the stimulation of the proliferation of chondrocytes. Overloaded pressure on cartilage and inflammatory factors such as interleukin (IL)-1, tumor necrosis factor (TNF)- $\alpha$ strike this balance (11). During the process of the development and progression of osteoarthritis (OA) or rheumatoid arthritis (RA), there is often initial destruction of superficial aggrecan by two predominant factors, ADAMTS-4 and -5 (12).

Although an increasing number of studies have indicated that OPG protects cartilage in vivo, whether OPG affects chondrocytes directly and the underlying cellular and molecular mechanisms of this effect remain to be established. The aim of the present study was to investigate the direct effect of OPG on chondrocyte viability and proliferation and the functional consequences of OPG treatment.

\section{Materials and methods}

Reagents. Dulbecco's modified Eagle's medium (DMEM) and type II collagenase were purchased from Gibco-BRL (Carlsbad, CA, USA), and fetal bovine serum (FBS) and trypsin-EDTA were purchased from HyClone (Logan, UT, USA). Cell Counting kit-8 (CCK-8) was purchased from Dojindo Laboratories (Kumamoto, Japan), and 96-well culture plates and $25-\mathrm{cm}^{2}$ flasks were purchased from Axygen (San Diego, CA, USA). Primer synthesis was performed by Shanghai Sangon Biological Engineering Technology Services, Ltd. (Shanghai, China). The SYBR-Green Real-Time PCR Master mix and TRIzol reagent were purchased from Takara (Shiga, Japan). RevertAid First Strand cDNA Synthesis kit was purchased from Fermentas (Amherst, NY, USA). Immunodetection of cell signal proteins for western blot analysis was achieved using the antibodies: anti-mitogenactivated/extracellular signal-regulated kinase kinase (MEK), anti-extracellular signal-regulated kinase (ERK)1/2, anti-P38 mitogen-activated protein kinase (P38MAPK), anti-c-Jun $\mathrm{N}$-terminal protein kinase (JNK), anti-NF- $\kappa \mathrm{Bp} 65$, anti-phospho (p) MEK, anti-p-ERK1/2, anti-p-P38MAPK, anti-p-JNK anti-p-NF- $\kappa$ Bp65 and anti-glyceraldehyde-3-phosphate dehydrogenate (GAPDH) from (Cell Signaling Technology, Inc., Beverly, MA, USA). In addition, rabbit anti-rat TIMP-4 and rabbit anti-rat ADAMTS-5 were purchased from Abcam (Cambridge, UK) and mouse anti-rat BMP-2 was purchased from Pierce (Madison, WI, USA). U0126 (a MEK inhibitor) and PD098059 (an ERK inhibitor) were purchased from Promega (Madison, WI, USA). The P38MAPK inhibitor, SB203580, was purchased from Merck Millipore (Billerica, MA, USA). PhosStop tablets were purchased from Roche (Basel, Switzerland). Recombinant human OPG was purchased from R\&D Systems (Minneapolis, MN, USA). Toluidine blue was provided by Sigma-Aldrich (St. Louis, MO, USA), and 5-(and-6)-carboxyfluorescein diacetate succinimidyl ester (CFSE) was provided by Invitrogen Life Technologies (Carlsbad, CA, USA).

Animals. Twelve 1-week-old, six 12-week-old, and six 36-week-old specific pathogen-free (SPF) Sprague-Dawley (SD) rats used in this study were purchased from the Zhejiang Academy of Medical Sciences (Huangzhou, China). Animals were fed and treated in accordance with the Guidelines for the Care and Use of Laboratory Animals 2006, administered by the First Affiliated Hospital of College of Medicine, Zhejiang University (Hangzhou, China).

Culture and identification of chondrocytes. The rats were sacrificed by cervical dislocation, soaked in $75 \%$ alcohol for $10 \mathrm{~min}$ and their knee joints were then separated and the superficial cartilage was removed by a sterile surgical knife on the clean bench. The cartilage was washed five times with ice-cold phosphate-buffered saline (PBS) containing 1\% ampicillin and streptomycin. Subsequent to this, the cartilage was sectioned into several $1-\mathrm{mm}^{3}$ slices which were placed on a plate containing $0.2 \%$ type II collagenase and transferred to a $37^{\circ} \mathrm{C}$ incubator. Supernatant was collected every $60 \mathrm{~min}$ and centrifuged at $250 \mathrm{x}$ g for $5 \mathrm{~min}$ to collect the cell pellet. These procedures were repeated four times. The cells were re-suspended in DMEM complete culture medium (containing $10 \% \mathrm{FBS}, 100 \mathrm{U} / \mathrm{ml}$ ampicillin and $100 \mathrm{U} / \mathrm{ml}$ streptomycin). Cells were filtered through 200 mesh stainless steel filters and seeded in flasks at a density of $1 \times 10^{5} / \mathrm{cm}^{2}$ and cultured at $37^{\circ} \mathrm{C}$ in a $5 \% \mathrm{CO}_{2}$ incubator. When the cells reached $80 \%$ confluence, the primary cultured cells were passaged. Chondrocytes were identified with toluidine blue staining, as described previously (13).

Treatment and inhibition. The cells were seeded in $35 \mathrm{~mm}^{2}$-wells at a density of $1 \times 10^{5}$ cells $/ \mathrm{cm}^{2}$. Following $24 \mathrm{~h}$ of starvation, the chondrocytes were treated with OPG at concentrations of 5, 10, 25, 50, 100 and $200 \mathrm{ng} / \mathrm{ml}$. The 
Table I. Primer sequences.

\begin{tabular}{lll}
\hline Gene & \multicolumn{1}{c}{ Sense $\left(5^{\prime} \rightarrow 3^{\prime}\right)$} & Antisense $\left(5^{\prime} \rightarrow 3^{\prime}\right)$ \\
\hline IGF-I & cttttacttcaacaagccaca & tacatctccagcctcctcaga \\
TGF- $\beta$ & tggaagtggatccacgcgcccagg & gcaggagcgcacgatcatgttggac \\
bFGF & tactgcaagaacggcggcttcttc & tactggccagttcgtttcagtgcc \\
BMP-2 & ggaaacttccegacgcttct & cctgcatttgttcccgaaaa \\
TIMP-1 & tggcatcctcttgttgctatc & cgaatcctttgagcatcttagtc \\
TIMP-2 & aacccatcaagaggattcaa & cagggcacaataaagtcacaga \\
TIMP-3 & gcgtgtatgaaggcaagatgta & gcgtagtgtttggactgatagc \\
TIMP-4 & cgtctgccactctgctttagta & ctgcttctgactgttggtttct \\
ADAMTS-4 & cccggaatggtggaagtatt & tcttcacggaaggtcaatgct \\
ADAMTS-5 & ctacagcaactccgtgtgtgtc & agtctggtctttggctttgaac \\
Collagen II & ctcaagtcgctgaacaacc & ctatgtccacaccaaattcc \\
Aggrecan & aggatggcttccaccagtgc & tgcgtaaaagacctcaccctcc \\
GADPH & acagcaacagggtggtggac & tttgagggtgcagcgaactt
\end{tabular}

IGF-I, insulin-like growth factor-I; TGF- $\beta$, transforming growth factor- $\beta$; bFGF, basic fibroblast growth factor; BMP-2, bone morphogenetic protein-2; TIMP, tissue inhibitor of metalloproteinase; GADPH, glyceraldehyde-3-phosphate dehydrogenate.

maximum effective dose was selected and used for the rest of the experiments. RNA was extracted for reverse transcription-polymerase chain reaction (RT-PCR) analysis after $24 \mathrm{~h}$ of OPG treatment and protein for western blot analysis after $48 \mathrm{~h}$ of treatment. Chondrocytes were incubated with various inhibitors for $30 \mathrm{~min}$ prior to the addition of OPG. PD098059 was used at $10 \mu \mathrm{M}, \mathrm{U} 0126$ at $5 \mu \mathrm{M}$ and SB203580 at $20 \mu \mathrm{M}$.

Assessment of cell proliferation. Passage 1 chondrocytes were plated at a density of $5 \times 10^{3}$ cells/well in a 96 -well plate in $0.1 \mathrm{ml}$ DMEM complete culture medium. The cells were cultured for $24 \mathrm{~h}$ and then starved for $24 \mathrm{~h}$ in serum-free DMEM. The chondrocytes were incubated with or without U0126 (a MEK inhibitor), PD098059 (an ERK inhibitor) or SB203580 (a P38MAPK inhibitor). After $30 \mathrm{~min}, \mathrm{OPG}$ was added (final concentrations of 5, 10, 25, 50, 100, 200 and $500 \mathrm{ng} / \mathrm{ml}$ ) and the cells were incubated for an additional $48 \mathrm{~h}$ at $37^{\circ} \mathrm{C}$. The cells were then counted using CCK-8. Briefly, $10 \mu \mathrm{l}$ of the kit reagent was added to each well and the cells were incubated for $2 \mathrm{~h}$. Cell viability was determined by measuring the absorbance at 450 and $655 \mathrm{~nm}$ with a microplate reader (Microplate Reader 680; Bio-Rad Laboratories, Hercules, CA, USA). Each experimental condition was analyzed in five wells. Chondrocytes used were isolated from 1-, 12- and 36-week-old SD rats.

Western blot analysis detection of MEK, ERK1/2, P38MAPK, JNK, NF- BP 65, TIMP-4 and ADAMTS-5 in chondrocytes following OPG treatment. Subsequent to OPG stimulation for $0,15,30$ and $60 \mathrm{~min}$, the total protein was extracted from each group according to the manufacturer's instructions. For the determination of TIMP-4 and ADAMTS-5 the cells were incubated with OPG for 2 days. A bicinchoninic acid assay was used to determine the protein concentration. PhosStop was used to inhibit the degradation of phosphoprotein. Samples from different groups containing 20 or $30 \mu \mathrm{g}$ proteins were loaded onto a $10 \%$ sodium dodecyl sulfate-polyacrylamide gel, electrophoresed and transferred to a polyvinylidene fluoride (PVDF) membrane. Following blocking with 5\% non-fat milk, the PVDF membranes were incubated with primary antibodies in a Tris-buffered saline with Tween 20 (TBST) buffer overnight at $4^{\circ} \mathrm{C}$. The primary antibodies used were against p-MEK, MEK, p-ERK1/2, ERK1/2, p-P38MAPK, P38MAPK, p-JNK, JNK, p-NF-кBp65, NF-кBp65, TIMP-4 and ADAMTS-5. GAPDH served as an internal control. On the following day, subsequent to three washes with TBST, the membranes were incubated with the appropriate secondary antibodies for $1 \mathrm{~h}$ at room temperature. The membranes were washed another three times when the membranes had been soaked in an enhanced chemiluminescence reagent for $5 \mathrm{~min}$, and the bands were visualized with X-ray film (VersaDoc Imaging system; Bio-Rad, Hercules, CA, USA). The intensity of the bands was analyzed by Quantity One software (Bio-Rad). Data were expressed in arbitrary units.

qPCR detection for anabolic and catabolic factors expressed by chondrocytes. The total RNA in each group was extracted with TRIzol reagent according to the manufacturer's instructions. RNA $(1 \mu \mathrm{g})$ was reverse transcribed into cDNA with the RevertAid First Strand cDNA Synthesis kit. The obtained cDNA was amplified by qPCR by an ABI VII Real-Time PCR system (Applied Biosystems, Carlsbad, CA, USA) and SYBR-Green Real-Time PCR Master mix. The primers used for qPCR are listed in Table I, with GAPDH serving as an internal control. The conditions for the $\mathrm{qPCR}$ amplification reaction were $95^{\circ} \mathrm{C}$ for $10 \mathrm{sec}, 95^{\circ} \mathrm{C}$ for $30 \mathrm{sec}, 60^{\circ} \mathrm{C}$ for $40 \mathrm{sec}$ and $72^{\circ} \mathrm{C}$ for $45 \mathrm{sec}$, for a total of 40 cycles. The dissolution curve was analyzed to determine the specificity of the qPCR amplification. Quantification of the relative expression levels of the target genes was achieved using the formula: $2^{-\Delta \Delta \mathrm{Ct}}$, where $\Delta \Delta \mathrm{Ct}=(\mathrm{Ct}$ of the target gene $-\mathrm{Ct}$ of GAPDH $) \times$ treatment - (Ct of the target gene - $\mathrm{Ct}$ of GAPDH) $\mathrm{x}$ control. Data were presented in arbitrary units relative to the control, which was defined as a value of 1 . 

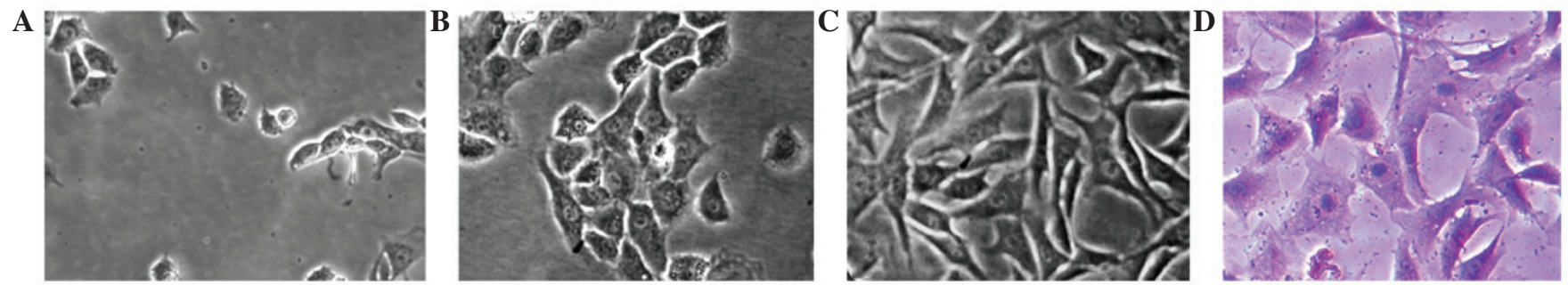

Figure 1. Growth and identification of chondrocytes. (A) The primary chondrocytes began to adhere and spread out to form pseudopods after $~ 24 \mathrm{~h}$ of culture. (B) The primary chondrocytes were oval-shaped and clustered together into a paving stone-like arrangement after 3-4 days culture. (C) Passage 1 chondrocytes were trilateral or polygonal in shape with more pseudopods. (D) Toluidine blue staining shows dark blue nuclei and purple metachromatic granules within and around the chondrocytes.

Detection of cell proliferation by CFSE using fluorescence-activated cell sorting (FACS). Passage 1 chondrocytes were collected and incubated with CFSE for $15 \mathrm{~min}$ at $37^{\circ} \mathrm{C}$ in the dark, and were then placed in cold DMEM containing $10 \%$ ice-cold FBS. The cells were seeded in 6-well plates (density, $1 \times 10^{5}$ cells/well). When the cells had been cultured for $24 \mathrm{~h}$, they were treated with or without OPG for 4 days in an incubator. The cells were then digested with $0.25 \%$ trypsin, collected and suspended in FACS buffer. For CFSE detection, the cells were analyzed directly by FACS. The results were interpreted by FlowJo (Tree Star, Inc., Ashland, OR, USA).

Statistical analysis. Data were expressed as the mean \pm SEM. Statistical significance was assessed by a two-tailed Student's t-test. $\mathrm{P}<0.05$ was considered to indicate a statistically significant difference.

\section{Results}

Growth and identification of chondrocytes. Chondrocytes were small and round when initially seeded, and began to adhere and gradually spread out to form pseudopods after $\sim 24 \mathrm{~h}$ (Fig. 1A). With an appropriate seeding density of $1 \times 10^{5} / \mathrm{cm}^{2}$ in the present study, the cells grew in clusters and completely covered the culture flask within 3-4 days. The primary chondrocytes were oval and formed paving stone-like arrangement (Fig. 1B) and the passage 1 chondrocytes were trilateral or polygonal with more pseudopods (Fig. 1C). The chondrocytes were identified by toluidine blue staining and purple metachromatic granules were observed within and around the cells (Fig. 1D).

Effect of OPG on chondrocyte viability and proliferation. Cell viability and proliferation were analyzed by CCK-8. To investigate the effect of OPG on the number of chondrocytes, chondrocytes were treated with increasing doses of OPG. As demonstrated by Kotake et al (14), OPG concentrations were $5-35 \mathrm{ng} / \mathrm{ml}$ in the synovial fluid. The cartilage was surrounded by the synovial fluid, thus chondrocytes are exposed to an in vitro concentration range of $5-200 \mathrm{ng} / \mathrm{ml}$ OPG. OPG at a concentration of $10 \mathrm{ng} / \mathrm{ml}$ exerted the maximum effect on the proliferation of chondrocytes (Fig. 2).

$O P G$-induced chondrocyte proliferation through the $M E K / E R K$ signaling pathway. The effect of OPG was analyzed on the phosphorylation of three MAPKs, ERK, P38MAPK and

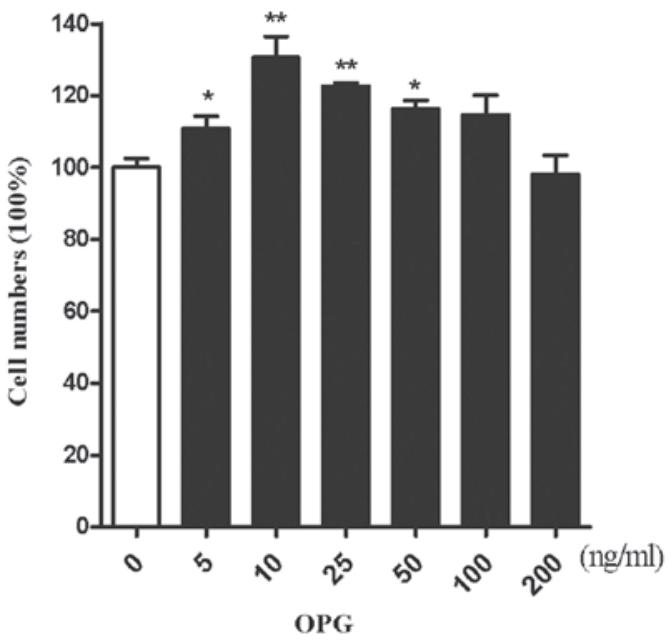

Figure 2. Effect of OPG on chondrocyte viability and proliferation analyzed by a CCK-8 assay. Chondrocytes were incubated with OPG at various concerntrations of 5,10,25,50,100 and $200 \mathrm{ng} / \mathrm{ml}$. Columns show the percentage of the values obtained from cells without treatment. Data are presented as the mean \pm SEM of four independent wells. ${ }^{*} \mathrm{P}<0.05$ and ${ }^{* *} \mathrm{P}<0.01$, vs. the control group. OPG, osteoprotegerin; CCK-8, Cell Counting kit-8.

JNK. Phosphorylation of ERK1/2 was increased to 4.12-fold that of untreated samples at $15 \mathrm{~min}$ and 2.12-fold at $30 \mathrm{~min}$ following treatment with OPG. However, P38MAPK and JNK signaling were not influenced by OPG during the treatment period (Fig. 3A-C).

The upstream and downstream signaling of ERK1/2 signaling pathways was then analyzed in the chondrocytes. As confirmed by previous studies, extracellular signals are transmitted by MEK1/2 in ERK1/2 signaling cascades (15-17). To elucidate the ERK1/2 signaling cascades involved in OPG-induced proliferation, the phosphorylation of MEK was analyzed simultaneously with ERK1/2. The $10 \mathrm{ng} / \mathrm{ml}$ concentration was selected to be used in all experiments based on the CCK-8 assay. As shown in Fig. 3D, the level of p-MEK was 3.5- and 3.0-fold higher in the OPG 15 and 30 min groups, respectively, than in the control group (Fig. 3D).

Previous studies have demonstrated that NF- $\mathrm{\kappa B}$ is involved in the proliferation of tumor and other cells (18-21), and acts as a downstream effector of ERK1/2. Thus, ERK1/2 signaling was analyzed by determining the phosphorylation of ERK1/2 and NF- $\kappa$ B simultaneously. As shown in Fig. 3F, the NF- $\kappa B$ phosphorylation level was not affected by OPG stimulation during the exposure times used (Fig. 3F). 

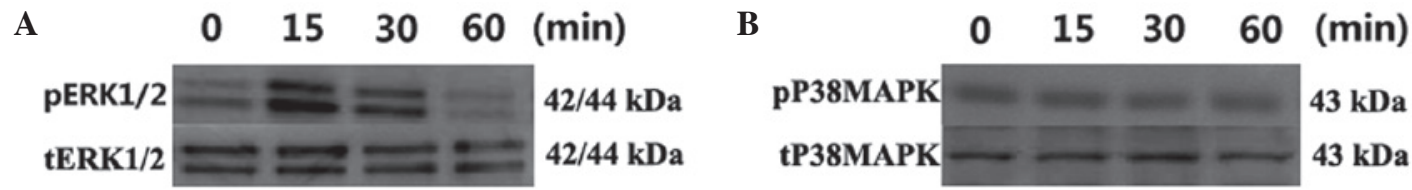

C

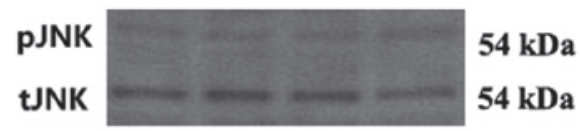

$\mathbf{E}$

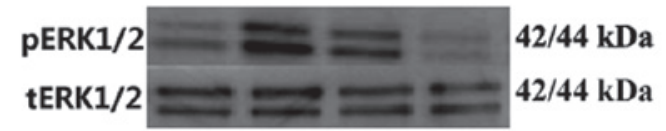

D

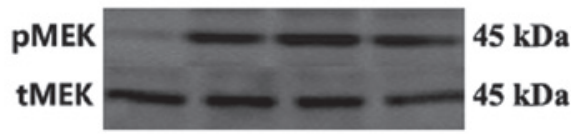

$\mathbf{F}$



Figure 3. Effect of OPG on MEK, ERK1/2, P38MAPK, JNK and NF-кBp65 phosphorylation analyzed by western blot analysis. Phosphorylation of the MAPK family following treatment: (A) ERK1/2; (B) P38MAPK and (C) JNK. Phosphorylation of the MEK/ERK/NF- $\mathrm{KB}$ cascade: (D) MEK, (E) ERK1/2 and (F) NF- $\mathrm{kBp} 65$. Chondrocytes were treated with $10 \mathrm{ng} / \mathrm{ml} \mathrm{OPG}$ for 0, 15, 30 and $60 \mathrm{~min}$. The results shown represent three independent experiments. OPG, osteoprotegerin; MEK, mitogen-activated/extracellular signal-regulated kinase kinase; ERK, extracellular signal-regulated kinase; P38MAPK, P38

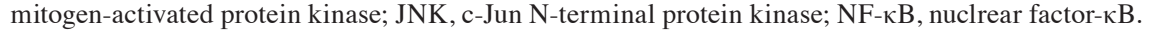

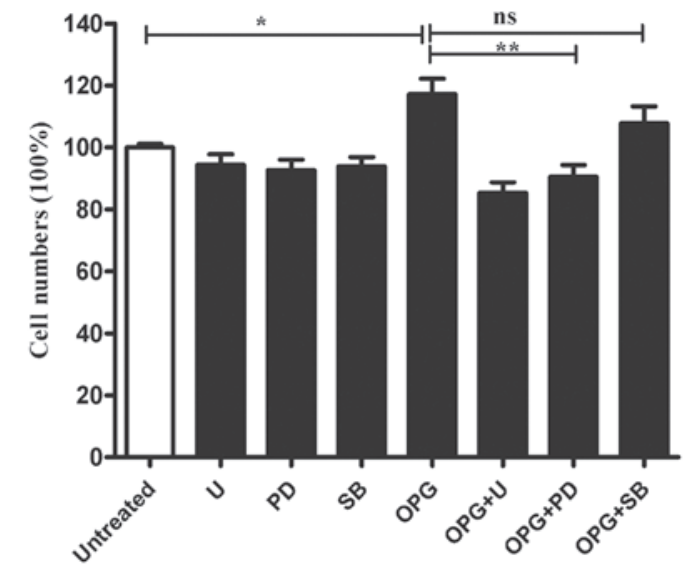

Figure 4. Inhibition of MEK or ERK rather than P38MAPK activation suppressed OPG-mediated chondrocyte proliferation. Relative cell proliferation was analyzed by CCK-8. Columns show the percentage of the values obtained from cells without treatment. Untreated, treated without OPG; $\mathrm{U}$, cells treated with $5 \mu \mathrm{M}$ U0126 (MEK inhibitor); PD, cells treated with $10 \mu \mathrm{M}$ PD098095 (ERK inhibitor); OPG, cells treated with $10 \mathrm{ng} / \mathrm{ml}$ OPG; $\mathrm{OPG}+\mathrm{U}, \mathrm{OPG}+\mathrm{PD}, \mathrm{OPG}+\mathrm{SB}$, cells pretreated with the indicated concentration of U0126, PD098095, SB203580, then treated with $10 \mathrm{ng} / \mathrm{ml}$ OPG. MEK, mitogen-activated/extracellular signal-regulated kinase kinase; ERK, extracellular signal-regulated kinase; P38MAPK, P38 mitogen-activated protein kinase; OPG, osteoprotegerin; CCK-8, Cell Counting kit-8; ns, not significant. Data are presented as the mean \pm SEM of four independent wells value. ${ }^{*} \mathrm{P}<0.05$ and ${ }^{* *} \mathrm{P}<0.01$ vs. the control group.

Inhibition of OPG-induces chondrocyte proliferation following ERK activity suppression. To further determine whether the MAPK pathway was involved in the OPG-induced proliferation of chondrocytes. Chondrocytes were pretreated with U0126 (a MEK inhibitor), PD098059 (an ERK inhibitor) or SB203580 (a P38MAPK inhibitor) for $30 \mathrm{~min}$ prior to OPG stimulation. As shown in Fig. 4, when incubated with U0126 or PD98067, cell proliferation induced by OPG was suppressed significantly. While SB203580 only exhibited marginal influence on the cell proliferation induced by OPG (Fig. 4).



C



Figure 5. CFSE test for chondrocyte proliferation induced by OPG. The data show the percentage of resting chondrocytes not undergoing proliferation in (A) the control and (B) OPG groups. (C) Columns show the percentage of resting chondrocytes not undergoing proliferation. Data are presented as the mean \pm SEM of three independent experiments. ${ }^{*} \mathrm{P}<0.05$ vs. the control group. CFSE, 5-(and-6)-carboxyfluorescein diacetate succinimidyl ester; OPG, osteoprotegerin.

Effect of OPG on chondrocyte proliferation detected by CFSE. Following confirmation of the effect of OPG on chondrocyte proliferation by CCK- 8 , the proliferation effect was analyzed in greater detail using CFSE. While CCK- 8 only reflects the number of live cells, CFSE remains in the cell even when the cells have died or undergone apoptosis. Thus, CFSE shows a comprehensive profile of the cell proliferation. Consistent with the result of the CCK- 8 assay, OPG at the concentration of $10 \mathrm{ng} / \mathrm{ml}$ increases the proliferation rate of chondrocytes, with 75.9 and $67.2 \%$ of the cells undergoing proliferation in the OPG and control groups (Fig. 5), respectively. 
A

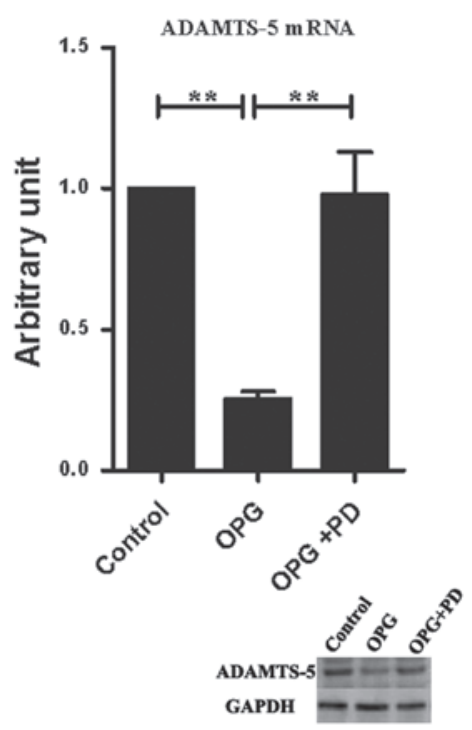

C

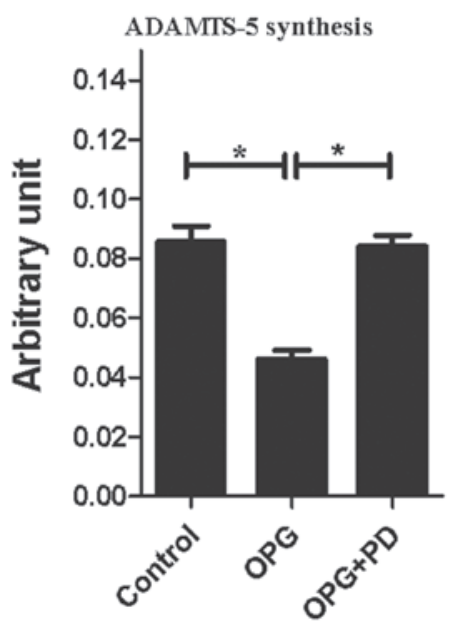

B

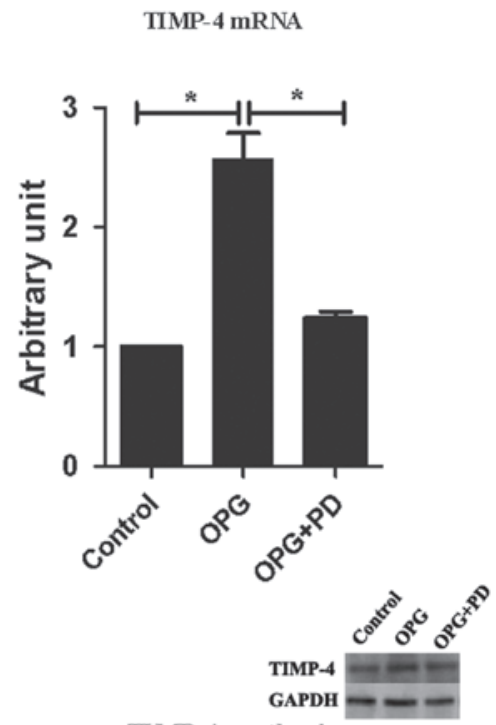

D

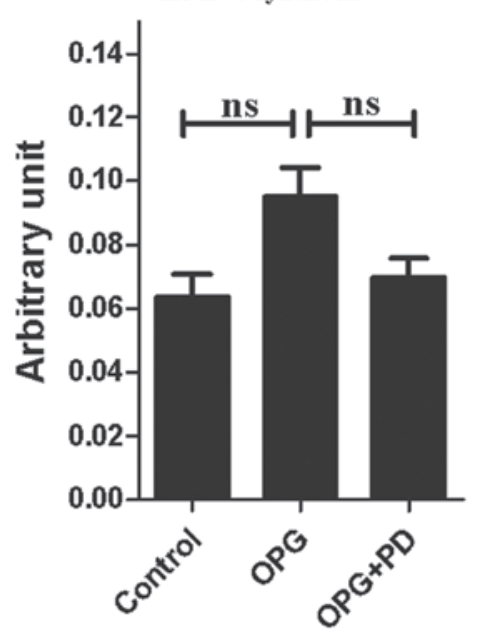

Figure 6. Alterations of expression levels of the catabolic and anabolic factors following OPG treatment. Gene expression level of (A) ADAMTS-5 and (B) TIMP-4, and protein production of (C) ADAMTS-5 and (D) TIMP-4 on chondrocytes. Control, untreated cells; OPG, cells treated with 10 ng/ml OPG; OPG + PD, cells pretreated with the indicated concentration of PD098095, then treated with $10 \mathrm{ng} / \mathrm{ml} \mathrm{OPG}$. The gene expression level is presented as arbitrary units compared with the control, which was attributed a value of 1 . The protein production is presented as arbitrary units compared to the internal control (GAPDH). The western blot analysis figures shown represents three independent experiments. Data are presented as the mean \pm SEM of three independent experiments. "P<0.05 and ${ }^{* *} \mathrm{P}<0.01$; vs. the control group. OPG, osteoprotegerin; TIMP-4, tissue inhibitor of metalloproteinase-4; GAPDH, glyceraldehyde 3-phosphate dehydrogenase; ns, not significant.

Alterations of expression levels of catabolic and anabolic factors following OPG treatment. Komuro et al (3) demonstrated that RANKL has no effect on the expression of proinflammatory mediators, including IL-1 $\beta$, IL-6, cyclooxygenase- 2 , TNF- $\alpha$, RANTES or inducible nitric oxide synthase, and collagenase activity was not affected by the treatment of OPG and RANKL. Kwan Tat et al (8) analyzed other factors, including IL-1 $\beta$, IL-6, IL-17, TNF- $\alpha$, MMP-1, -2, -9, and -13, TIMP-1 and PAR-2, and observed that OPG significantly promoted the expression of MMP-13 and PAR-2. Thus, in the present study, the gene expression levels of the anabolic factors containing TIMP-1, -2, -3 and -4 , IGF-I, TGF- $\beta$, bFGF, and BMP-2, as well as catabolic factors, including ADAMTS-4, ADAMTS-5 and constitutively expressed factors collagen II $\alpha 1$ and aggrecan were analyzed. OPG treatment significantly downregulated the gene expression of ADAMTS-5 (Fig. 6A) and upregulated the gene expression level of TIMP-4 (Fig. 6B). None of the other factors analyzed were affected by OPG treatment (data not shown).
The protein production of TIMP-4 and ADAMTS-5 were also analyzed following treatment with OPG. Subsequent to OPG treatment $48 \mathrm{~h}$, ADAMST-5 protein production exhibited the same profile of gene expression: 2.2-fold greater in the OPG group than in the control group, which was statistically significant (Fig. 6C). TIMP-4 production was 1.5-fold higher in the OPG group than the control group, while it did not reach statistical significance $(\mathrm{P}=0.55$ between the $\mathrm{OPG}$ and control groups, $\mathrm{P}=0.08$ between the $\mathrm{OPG}$ and $\mathrm{OPG}+\mathrm{PD}$ groups; Fig. 6D).

Inhibition of ERK1/2 activation by PD098095, suppressed OPG-mediated alteration of ADAMTS-5 and TIMP-4 expression of the gene and protein levels.

\section{Discussion}

OPG is predominantly expressed by osteoblasts, and its involvement in inhibiting osteoclastogenesis has been well 
documented; however, there are few studies concerning the effects of OPG on cartilage. In an OA mouse model, treatment with OPG exhibited a beneficial effect against the progression of cartilage destruction $(2,5)$, but the exact mechanism has not been fully elucidated. Moreover, little is known about the direct effect of OPG on chondrocytes. The present study suggests that OPG exerted a direct effect on chondrocytes, and it upregulated the expression of TIMP-4 and downregulated the expression of ADAMTS-5 (aggrecanase-2). This is partly consistent with a previous study, in which the number of ADAMTS-4 and -5 expressing cells was decreased by OPG treatment in an OA mouse model (5). The two aggrecanases have been identified to be essential factors in aggrecanolysis and cartilage destruction (12). It was demonstrated that ADAMTS-5 was predominant, and ADAMTS-5-knockout mice were protected against cartilage destruction in an unstable joint model (22). In the present study, ADAMTS-5 was decreased by $\sim 5$-fold in RNA expression and 2.2-fold in protein secretion. The expression profile of ADAMTS-4 was not changed by OPG treatment. Moreover, TIMP-4, an inhibitor of MMPs, was upregulated by OPG stimulation. However, Kwan Tat et al (8) demonstrated that OPG increased the gene expression level of MMP-13 and PAR-2, which were two catabolic factors in cartilage. This may be attributable to the different source of chondrocytes used in the studies, as OA chondrocytes were used in the previous study and normal chondrocytes were used in the present study. Alternatively, OPG changes the expression profile of catabolic and anabolic factors, with complex effects on the chondrocytes. In brief, the results of the present study aid in the understanding of OPG protection of cartilage in RA and OA mouse models. Other factors expressed by chondrocytes were not affected by OPG treatment.

Initially, the number of chondrocytes isolated from 1-weekold rats was increased by OPG treatment, as confirmed by a CCK-8 assay. It was investigated whether there was any difference among the effects of OPG on chondrocytes isolated from different aged rats. Therefore, chondrocytes isolated from 12- and 36-week-old rats were used to perform CCK-8 tests; however, no difference was observed (data not shown). This finding suggests OPG has a permanent and stable effect on chondrocytes as long as OPG within an appropriate concentration range is used.

There are a large number of signaling pathways that participate in the biological activity and behavior of chondrocytes. Signaling via JNK (23), phosphoinositol 3-kinase (PI3k)/AKT (24), Src (25), Wnt/ $\beta$-catenin (26) is important in maintaining the self-renewal of cartilage. MAPK signaling was regarded as important in varied cell responses, such as the proliferation, differentiation and apoptosis of chondrocytes. ERK1/2 and P38MAPK pathways have been confirmed to be involved in the cell proliferation and differentiation of chondrocytes (27-29). It was demonstrated that soluble OPG activated several signals in different cell types, inducing cytoskeleton reorganization through FAK, Src and ERK1/2 signaling in endothelial cells (1), promoting endothelial cell proliferation and migration partly via ERK1/2 signaling (30), increasing periodontal ligament cells expressing osteopontin via syndecan-1 and PI3k/AKT (9), altering the morphology and function of pancreatic islets possibly via the renin-angio- tensin system (31) and inducing proliferation of rodent vascular smooth muscle cells (32). In the present study, MAPK signaling molecules were analyzed as candidate downstream effectors of OPG. Accumulation of phosphorylation ERK1/2 is observed following OPG treatment. It was assumed that $\mathrm{NF}-\kappa \mathrm{B}$ was the downstream effector of ERK1/2, as NF- $\kappa \mathrm{B}$ participated in the differentiation of chondrocytes (33). However, the NF- $\kappa \mathrm{B}$ phosphorylation level was not affected by OPG stimulation. The ERK1/2 cascade was also investigated. MEK, one of the upstream factors of ERK1/2, was analyzed simultaneously with ERK1/2 and was activated by OPG treatment. Data collectively demonstrated that OPG promoted chondrocyte proliferation through the MEK/ERK pathway but not P38MAPK and JNK, and it was independent of NF- $\kappa$ B.

In conclusion, OPG exerted a direct effect on chondrocytes. It increased the expression of TIMP-4 and decreased the expression of ADAMTS-5. Moreover, it promoted the proliferation of chondrocytes through the MEK/ERK pathway, independent of the activation of NF- $\kappa \mathrm{B}$.

\section{Acknowledgements}

This study was supported by the Natural Science Fund of Ningbo Science and Technology Bureau (grant no. 201101A6110108).

\section{References}

1. Kobayashi-Sakamoto M, Isogai E and Holen I: Osteoprotegerin induces cytoskeletal reorganization and activates FAK, Src, and ERK signaling in endothelial cells. Eur J Haematol 85: 26-35, 2010.

2. Kadri A, Ea HK, Bazille C, Hannouche D, Lioté F and Cohen-Solal ME: Osteoprotegerin inhibits cartilage degradation through an effect on trabecular bone in murine experimental osteoarthritis. Arthritis Rheum 58: 2379-2386, 2008.

3. Komuro H, Olee T, Kühn K, et al: The osteoprotegerin/receptor activator of nuclear factor kappaB/receptor activator of nuclear factor kappaB ligand system in cartilage. Arthritis Rheum 44: 2768-2776, 2001.

4. Kong YY, Yoshida H, Sarosi I, et al: OPGL is a key regulator of osteoclastogenesis, lymphocyte development and lymph-node organogenesis. Nature 397: 315-323, 1999.

5. Shimizu S, Asou Y, Itoh S, et al: Prevention of cartilage destruction with intraarticular osteoclastogenesis inhibitory factor/osteoprotegerin in a murine model of osteoarthritis. Arthritis Rheum 56: 3358-3365, 2007.

6. Kong YY, Feige U, Sarosi I, et al: Activated T cells regulate bone loss and joint destruction in adjuvant arthritis through osteoprotegerin ligand. Nature 402: 304-309, 1999.

7. Kwan Tat S, Pelletier JP, Lajeunesse D, Fahmi H, Lavigne M and Martel-Pelletier J: The differential expression of osteoprotegerin (OPG) and receptor activator of nuclear factor kappaB ligand (RANKL) in human osteoarthritic subchondral bone osteoblasts is an indicator of the metabolic state of these disease cells. Clin Exp Rheumatol 26: 295-304, 2008.

8. Kwan Tat S, Amiable N, Pelletier JP, et al: Modulation of OPG, RANK and RANKL by human chondrocytes and their implication during osteoarthritis. Rheumatology (Oxford) 48: 1482-1490, 2009.

9. Yongchaitrakul T, Manokawinchoke J and Pavasant P: Osteoprotegerin induces osteopontin via syndecan-1 and phosphoinositol 3-kinase/Akt in human periodontal ligament cells J Periodontal Res 44: 776-783, 2009.

10. Goldring MB and Marcu KB: Cartilage homeostasis in health and rheumatic diseases. Arthritis Res Ther 11: 224, 2009.

11. Lories RJ and Luyten FP: The bone-cartilage unit in osteoarthritis. Nat Rev Rheumatol 7: 43-49, 2011.

12. Verma P and Dalal K: ADAMTS-4 and ADAMTS-5: key enzymes in osteoarthritis. J Cell Biochem 112: 3507-3514, 2011.

13. Li X, Peng J, Wu M, et al: BMP2 promotes chondrocyte proliferation via the $W n t / \beta$-catenin signaling pathway. Mol Med Rep 4: 621-626, 2011. 
14. Kotake S, Udagawa N, Hakoda M, et al: Activated human T cells directly induce osteoclastogenesis from human monocytes: possible role of $\mathrm{T}$ cells in bone destruction in rheumatoid arthritis patients. Arthritis Rheum 44: 1003-1012, 2001.

15. Rubinfeld $\mathrm{H}$ and Seger R: The ERK cascade: a prototype of MAPK signaling. Mol Biotechnol 31: 151-174, 2005.

16. Dai Y, Rahmani M, Pei XY, et al: Farnesyltransferase inhibitors interact synergistically with the Chk1 inhibitor UCN-01 to induce apoptosis in human leukemia cells through interruption of both Akt and MEK/ERK pathways and activation of SEK1/JNK. Blood 105: 1706-1716, 2005.

17. Kimata M, Michigami T, Tachikawa K, et al: Signaling of extracellular inorganic phosphate up-regulates cyclin D1 expression in proliferating chondrocytes via the $\mathrm{Na}^{+} / \mathrm{Pi}$ cotransporter Pit-1 and Raf/MEK/ERK pathway. Bone 47: 938-947, 2010.

18. Feng JQ, Xing L, Zhang JH, et al: NF-kappaB specifically activates BMP-2 gene expression in growth plate chondrocytes in vivo and in a chondrocyte cell line in vitro. J Biol Chem 278 29130-29135, 2003.

19. Sun HZ, Yang TW, Zang WJ and Wu SF: Dehydroepiandrosterone-induced proliferation of prostatic epithelial cell is mediated by NFKB via PI3K/AKT signaling pathway. J Endocrinol 204: 311-318, 2010.

20. Liu S, Tan WY, Chen QR, et al: Daintain/AIF-1 promotes breast cancer proliferation via activation of the NF-kappaB/cyclin D1 pathway and facilitates tumor growth. Cancer Sci 99: 952-957, 2008.

21. Massoumi R, Chmielarska K, Hennecke K, Pfeifer A and Fässler R: Cyld inhibits tumor cell proliferation by blocking Bcl-3-dependent NF-kappaB signaling. Cell 125: 665-677, 2006

22. Glasson SS, Askew R, Sheppard B, et al: Deletion of active ADAMTS5 prevents cartilage degradation in a murine model of osteoarthritis. Nature 434: 644-648, 2005.

23. Zhou Y, Millward-Sadler SJ, Lin H, et al: Evidence for JNK-dependent up-regulation of proteoglycan synthesis and for activation of JNK1 following cyclical mechanical stimulation in a human chondrocyte culture model. Osteoarthritis Cartilage 15: 884-893, 2007.
24. Kita K, Kimura T, Nakamura N, Yoshikawa H and Nakano T: $\mathrm{PI} 3 \mathrm{~K} / \mathrm{Akt}$ signaling as a key regulatory pathway for chondrocyte terminal differentiation. Genes Cells 13: 839-850, 2008

25. Bursell L, Woods A, James CG, Pala D, Leask A and Beier F: Src kinase inhibition promotes the chondrocyte phenotype. Arthritis Res Ther 9: R105, 2007.

26. Dong YF, Soung do Y, Chang Y, et al: Transforming growth factor-beta and Wnt signals regulate chondrocyte differentiation through Twist1 in a stage-specific manner. Mol Endocrinol 21: 2805-2820, 2007.

27. Ryan JA, Eisner EA, DuRaine G, You Z and Reddi AH: Mechanical compression of articular cartilage induces chondrocyte proliferation and inhibits proteoglycan synthesis by activation of the ERK pathway: implications for tissue engineering and regenerative medicine. J Tissue Eng Regen Med 3: 107-116, 2009.

28. Yonekura A, Osaki M, Hirota Y, et al: Transforming growth factor-beta stimulates articular chondrocyte cell growth through p44/42 MAP kinase (ERK) activation. Endocr J 46: 545-553, 1999.

29. Yosimichi G, Nakanishi T, Nishida T, Hattori T, TakanoYamamoto $\mathrm{T}$ and Takigawa M: CTGF/Hcs 24 induces chondrocyte differentiation through a p38 mitogen-activated protein kinase (p38MAPK), and proliferation through a p44/42 MAPK/extracellular-signal regulated kinase (ERK). Eur J Biochem 268: 6058-6065, 2001.

30. Kobayashi-Sakamoto M, Isogai E, Hirose K and Chiba I: Role of alphav integrin in osteoprotegerin-induced endothelial cell migration and proliferation. Microvasc Res 76: 139-144, 2008.

31. Toffoli B, Bernardi S, Candido R, et al: Osteoprotegerin induces morphological and functional alterations in mouse pancreatic islets. Mol Cell Endocrinol 331: 136-142, 2011.

32. Candido R, Toffoli B, Corallini F, et al: Human full-length osteoprotegerin induces the proliferation of rodent vascular smooth muscle cells both in vitro and in vivo. J Vasc Res 47: 252-261, 2010

33. Bradley EW and Drissi MH: WNT5A regulates chondrocyte differentiation through differential use of the CaN/NFAT and IKK/NF-kappaB pathways. Mol Endocrinol 24: 1581-1593, 2010. 